\section{Federation University ResearchOnline}

\section{https://researchonline.federation.edu.au}

Copyright Notice

This is the published version of the following article:

Gala, Sadek, Ragusa, Maria Alessandra, \& Théra, Michel. (2021). A new regularity criterion of weak solutions to the 3D micropolar fluid flows in terms of the pressure. Bollettino Della Unione Matematica Italiana (2008), 14(2), 331-337.

Copyright @ Bolletino dell Unione Matematica Italiana

This is the published version of the work. It is posted here with the permission of the publisher for your personal use. No further use or distribution is permitted.

https://doi.org/10.1007/s40574-020-00257-2 


\title{
A new regularity criterion of weak solutions to the 3D micropolar fluid flows in terms of the pressure
}

\author{
Sadek Gala ${ }^{1,2} \cdot$ Maria Alessandra Ragusa ${ }^{2,3}$ D Michel Théra $^{4,5}$
}

Received: 16 April 2020 / Accepted: 8 September 2020 / Published online: 21 September 2020

(c) Unione Matematica Italiana 2020

\section{Abstract}

In this study, we establish a new regularity criterion of weak solutions to the three-dimensional micropolar fluid flows by imposing a critical growth condition on the pressure field.

Keywords Micropolar fluid flows · Weak solutions · Pressure criterion · Besov spaces

Mathematics Subject Classification 35Q35 · 35B65 · 76D05

\section{Introduction and the main result}

In this paper we consider the following Cauchy problem for the incompressible micropolar fluid equations in $\mathbb{R}^{3}$ :

$$
\left\{\begin{array}{l}
\partial_{t} u-\Delta u+(u \cdot \nabla) u+\nabla \pi-\nabla \times \omega=0 \\
\partial_{t} \omega-\Delta \omega-\nabla \nabla \cdot \omega+2 \omega+(u \cdot \nabla) \omega-\nabla \times u=0 \\
\nabla \cdot u=0 \\
u(x, 0)=u_{0}(x), \omega(x, 0)=\omega_{0}(x)
\end{array}\right.
$$

where $u=u(x, t) \in \mathbb{R}^{3}, \omega=\omega(x, t) \in \mathbb{R}^{3}$ and $\pi=\pi(x, t)$ denote the unknown velocity of the fluid, the micro-rotational velocity of the fluid particles and the unknown scalar pressure of the fluid at the point $(x, t) \in \mathbb{R}^{3} \times(0, T)$, respectively, while $u_{0}, \omega_{0}$ are given initial data satisfying $\nabla \cdot u=0$ in the sense of distributions.

This model for micropolar fluid flows proposed by Eringen [6] enables to consider some physical phenomena that cannot be treated by the classical Navier-Stokes equations for the

Maria Alessandra Ragusa

maragusa@dmi.unict.it

1 University of Mostaganem, P. O. Box 270, 27000 Mostaganem, Algeria

2 Dipartimento di Matematica e Informatica, Università di Catania, Viale Andrea Doria, 6, 95125 Catania, Italy

3 RUDN University, 6 Miklukho-Maklay St, Moscow 117198, Russia

4 XLIM UMR-CNRS 7252 Université de Limoges, Limoges, France

5 Centre for Informatics and Applied Optimisation, Federation University Australia, Ballarat, Australia 
viscous incompressible fluids, such as for example, the motion of animal blood, muddy fluids, liquid crystals and dilute aqueous polymer solutions, colloidal suspensions, etc.

When the micro-rotation effects are neglected or $\omega=0,(1.1)$ reduces to the incompressible Navier-Stokes equations, and it is well known that the regularity criteria for weak solution for the fluid dynamical models attracts more and more attention. There are many important results on the velocity or vorticity or pressure blow-up criteria for Navier-Stokes equations, micropolar fluid equations and MHD equations and so on (see e.g., [1-4,7-10,14,16,17] and the references therein).

As for the pressure criterion, let us first recall some interesting results on pressure regularity of Navier-Stokes equations. In Ref. [13], He and Gala proved regularity of weak solutions under the condition

$$
\int_{0}^{T}\|\pi(\cdot, t)\|_{\dot{B}_{\infty, \infty}^{-1}}^{2} d t<\infty
$$

Here and thereafter, $\dot{B}_{\infty, \infty}^{-1}$ stands for the homogeneous Besov space, (for the definition see e.g. $[12,13])$. Later on, Guo and Gala [12] refined the condition (1.2) to

$$
\int_{0}^{T} \frac{\|\pi(\cdot, t)\|_{\dot{B}_{\infty, \infty}^{-1}}^{2}}{1+\log \left(e+\|\pi(\cdot, t)\|_{\dot{B}_{\infty, \infty}^{-1}}\right)} d t<\infty .
$$

Motivated by the paper of Guo and Gala [12], the aim of this paper is to give a new regularity criterion for weak solutions to the 3D micropolar fluid flows in terms of the pressure in critical Besov spaces. More precisely, our main result reads as follows.

Theorem 1.1 Let $T>0$ and $\left(u_{0}, \omega_{0}\right) \in L^{2}\left(\mathbb{R}^{3}\right) \cap L^{4}\left(\mathbb{R}^{3}\right)$ with $\nabla \cdot u_{0}=0$ in the sense of distributions. Assume that $(u, \omega)$ is a weak solution of the $3 D$ micropolar fluid flows (1.1) on $(0, T)$. If the pressure $\pi$ satisfies the following condition :

$$
\int_{0}^{T} \frac{\|\pi(\cdot, t)\|_{\dot{B}_{\infty, \infty}^{-1}}^{2}}{\left(e+\log \left(e+\|\pi(\cdot, t)\|_{\dot{B}_{\infty, \infty}^{-1}}\right)\right) \log \left(e+\log \left(e+\|\pi(\cdot, t)\|_{\dot{B}_{\infty, \infty}^{-1}}\right)\right)} d t<\infty,
$$

then $(u, \omega)$ is regular on $(0, T]$, i.e., $(u, \omega) \in C^{\infty}\left(\mathbb{R}^{3} \times(0, T]\right)$.

Remark 1.1 This result provides a new information concerning the question of the regularity of weak solutions of the micropolar fluid equations and extends those of [13] and [12]. In particular, the double-logarithm estimate (1.4) is sharper than any other results $[12,13]$.

Before stating our result, let us recall what we mean by a weak solution.

Definition 1.2 ([14]) Let $\left(u_{0}, \omega_{0}\right) \in L^{2}\left(\mathbb{R}^{3}\right)$ and $\nabla \cdot u_{0}=0$. A measurable function $(u(x, t), \omega(x, t))$ is called a weak solution to the $3 D$ micropolar flows equations (1.1) on $(0, T)$ if $(u, \omega)$ satisfies the following properties :

(1) $(u, \omega) \in L^{\infty}\left((0, T) ; L^{2}\left(\mathbb{R}^{3}\right)\right) \cap L^{2}\left((0, T) ; H^{1}\left(\mathbb{R}^{3}\right)\right)$ for all $T>0$;

(2) $(u(x, t), \omega(x, t))$ verifies $(1.1)$ in the sense of distribution;

(3) The following energy inequality holds :

$$
\begin{aligned}
& \|u(\cdot, t)\|_{L^{2}}^{2}+\|\omega(\cdot, t)\|_{L^{2}}^{2}+2 \int_{0}^{t}\left(\|\nabla u(\cdot, \tau)\|_{L^{2}}^{2}+\|\nabla \omega(\cdot, \tau)\|_{L^{2}}^{2}+\|\nabla \cdot \omega(\cdot, \tau)\|_{L^{2}}^{2}\right) d \tau \\
& \quad \leq\left\|u_{0}\right\|_{L^{2}}^{2}+\left\|\omega_{0}\right\|_{L^{2}}^{2},
\end{aligned}
$$

for all $0 \leq t \leq T$. 
By a strong solution we mean a weak solution $(u, \omega)$ such that

$$
(u, \omega) \in L^{\infty}\left((0, T) ; H^{1}\left(\mathbb{R}^{3}\right)\right) \cap L^{2}\left((0, T) ; H^{2}\left(\mathbb{R}^{3}\right)\right) .
$$

It is well known that strong solutions are regular (say, classical) and unique in the class of weak solutions.

In order to prove Theorem 1.1, we first establish some estimates between pressure and velocity. Taking div and $\nabla$ div to both sides of the micropolar fluid flows for smooth solution $(u, \pi)$, separately, we get the well-known pressure-velocity relation in $\mathbb{R}^{3}$, given by

$$
\pi=(-\Delta)^{-1} \sum_{i, j=1}^{3} \frac{\partial^{2}}{\partial x_{i} \partial x_{j}}\left(u_{i} u_{j}\right) \text { and } \nabla \pi=(-\Delta)^{-1} \sum_{i, j=1}^{3} \frac{\partial^{2}}{\partial x_{i} \partial x_{j}}\left(\nabla\left(u_{i} u_{j}\right)\right) .
$$

Then, the Calderón-Zygmund inequality implies that for any $1<\alpha<+\infty$ :

$$
\|\pi\|_{L^{\alpha}} \leq C\|u\|_{L^{2 \alpha}}^{2} \quad \text { and } \quad\|\nabla \pi\|_{L^{\alpha}} \leq C\||u| \nabla u\|_{L^{\alpha}} .
$$

\section{Proof of Theorem 1.1}

Now we are in the position to prove Theorem 1.1. Firstly, by means of the local existence result, which is similar to the one of the Navier-Stokes equations (refer to Giga [11], see also Dong et al. [5]), and the standard local solution extension technique, Eq. (1.1) with $\left(u_{0}, \omega_{0}\right) \in L^{2}\left(\mathbb{R}^{3}\right) \cap L^{4}\left(\mathbb{R}^{3}\right)$ admits a unique $L^{4}$-strong solution $(u, \omega)$ on a maximal time interval. For the notation simplicity, we may suppose that the maximal time interval is $[0, T)$. Thus, in order to prove Theorems 1.1, it remains to show that

$$
\lim _{t \rightarrow T}\left(\|u(t)\|_{4}+\|w(t)\|_{4}\right)<\infty .
$$

This will lead to a contradiction to the estimates to be derived below.

Proof Before going into the proof, we recall the following inequality established in Ref. [15] (see also [12]) :

$$
\|f\|_{L^{4}}^{2} \leq C\|f\|_{B_{\infty, \infty}^{-1}}\|\nabla f\|_{L^{2}} .
$$

Testing (1.1) 1 by $u|u|^{2}$ and using ( 1.1) 3 , we get

$$
\begin{aligned}
& \frac{1}{4} \frac{d}{d t}\|u\|_{L^{4}}^{4}+\||u| \nabla u\|_{L^{2}}^{2}+\frac{1}{2}\left\|\nabla|u|^{2}\right\|_{L^{2}}^{2} \\
& \quad=\int_{\mathbb{R}^{3}}(\nabla \times \omega) \cdot u|u|^{2} d x-\int_{\mathbb{R}^{3}}(u \cdot \nabla \pi)|u|^{2} d x \\
& \quad=\int_{\mathbb{R}^{3}} \omega\left[\nabla \times\left(u|u|^{2}\right)\right] d x+\int_{\mathbb{R}^{3}} \pi u \cdot \nabla|u|^{2} d x
\end{aligned}
$$

Testing (1.1) $)_{2}$ by $\omega|\omega|^{2}$ and using (1.1) $)_{3}$, we infer that

$$
\begin{aligned}
& \frac{1}{4} \frac{d}{d t}\|\omega\|_{L^{4}}^{4}+\||\omega| \nabla \cdot \omega\|_{L^{2}}^{2}+\frac{1}{2}\left\|\nabla|\omega|^{2}\right\|_{L^{2}}^{2} \\
& \quad=\int_{\mathbb{R}^{3}}(\nabla \times u) \cdot \omega|\omega|^{2} d x-2\|\omega\|_{L^{4}}^{4} \\
& \quad=\int_{\mathbb{R}^{3}} u\left[\nabla \times\left(\omega|\omega|^{2}\right) d x-2\|\omega\|_{L^{4}}^{4},\right.
\end{aligned}
$$


where we have used the following identities due to the divergence free property of the velocity field $u$ :

$$
\int_{\mathbb{R}^{3}}(u \cdot \nabla) u \cdot u|u|^{2} d x=0=\int_{\mathbb{R}^{3}}(u \cdot \nabla) \omega \cdot \omega|\omega|^{2} d x .
$$

Summing up (2.2) and (2.3), it follows that

$$
\begin{aligned}
& \frac{1}{4} \frac{d}{d t}\left(\|u\|_{L^{4}}^{4}+\|\omega\|_{L^{4}}^{4}\right)+\||u| \nabla u\|_{L^{2}}^{2}+\frac{1}{2}\left\|\nabla|u|^{2}\right\|_{L^{2}}^{2}+\||\omega| \nabla \cdot \omega\|_{L^{2}}^{2}+\frac{1}{2}\left\|\nabla|\omega|^{2}\right\|_{L^{2}}^{2} \\
& \quad=\int_{\mathbb{R}^{3}} \omega\left[\nabla \times\left(u|u|^{2}\right)\right] d x+\int_{\mathbb{R}^{3}} u\left[\nabla \times\left(\omega|\omega|^{2}\right) d x-2\|\omega\|_{L^{4}}^{4}+\int_{\mathbb{R}^{3}} \pi u \cdot \nabla|u|^{2} d x .\right.
\end{aligned}
$$

Using the Hölder inequality and the Young inequality and integration by parts, we derive the estimate of the first three terms on the right-hand side of (2.4) as follows:

$$
\begin{aligned}
& \int_{\mathbb{R}^{3}} \omega\left[\nabla \times\left(u|u|^{2}\right)\right] d x+\int_{\mathbb{R}^{3}} u\left[\nabla \times\left(\omega|\omega|^{2}\right) d x-2\|\omega\|_{L^{4}}^{4}\right. \\
& \quad \leq\|u\|_{L^{4}}\|\omega\|_{L^{4}}\left(\left\|\left|u\|\nabla u\|_{L^{2}}+\|\mid \omega\| \nabla \omega \|_{L^{2}}\right)-2\right\| \omega \|_{L^{4}}^{4}\right. \\
& \quad \leq\left\|\left|u\|\nabla u\|_{L^{2}}^{2}+\|\mid \omega\| \nabla \omega\left\|_{L^{2}}^{2}+C\right\| u \|_{L^{4}}^{4} .\right.\right.
\end{aligned}
$$

To estimate the last term of the right-hand side of (2.4), we have after integrating by parts and employing the Hölder inequality and the Young inequality,

$$
\begin{aligned}
\int_{\mathbb{R}^{3}} \pi u \cdot \nabla|u|^{2} d x & \leq\left.\int_{\mathbb{R}^{3}}|\pi||u||\nabla| u\right|^{2} \mid d x \\
& \leq C\|\pi\|_{L^{4}}^{2}\|u\|_{L^{4}}^{2}+\frac{1}{4}\left\|\nabla|u|^{2}\right\|_{L^{2}}^{2} \\
& \leq C\|\pi\|_{\dot{B}_{\infty, \infty}^{-1}}\|\nabla \pi\|_{L^{2}}\|u\|_{L^{4}}^{2}+\frac{1}{4}\left\|\nabla|u|^{2}\right\|_{L^{2}}^{2} \\
& \leq C\|\pi\|_{\dot{B}_{\infty, \infty}^{-1}}^{-1}\|u\|_{L^{4}}^{2}\||u| \nabla u\|_{L^{2}}+\frac{1}{4}\left\|\nabla|u|^{2}\right\|_{L^{2}}^{2} \\
& \leq C\|\pi\|_{\dot{B}_{\infty}^{-1}, \infty}^{2}\|u\|_{L^{4}}^{4}+\frac{1}{2}\left\|\left|u\|\nabla u\|_{L^{2}}^{2}+\frac{1}{4}\left\|\nabla|u|^{2}\right\|_{L^{2}}^{2}\right.\right.
\end{aligned}
$$

and hence

$$
\begin{aligned}
& \frac{d}{d t}\left(\|u(t)\|_{L^{4}}^{4}+\|\omega(t)\|_{L^{4}}^{4}\right)+\||u| \nabla u\|_{L^{2}}^{2}+\left\|\nabla|u|^{2}\right\|_{L^{2}}^{2}+\||\omega| \nabla \cdot \omega\|_{L^{2}}^{2}+\||\omega| \mid \nabla \omega\|_{L^{2}}^{2} \\
& \quad \leq C\left(1+\|\pi\|_{\dot{B}_{\infty, \infty}^{-1}}^{2}\right)\|u\|_{L^{4}}^{4}
\end{aligned}
$$

Taking the Gronwall inequality into consideration, one shows that

$$
\begin{aligned}
& \|u(\cdot, t)\|_{L^{4}}^{4}+\|\omega(\cdot, t)\|_{L^{4}}^{4} \\
& \quad \leq\left(\left\|u_{0}\right\|_{L^{4}}^{4}+\left\|\omega_{0}\right\|_{L^{4}}^{4}\right) \exp \left(C \int_{0}^{t}\left(1+\|\pi(\cdot, \tau)\|_{\dot{B}_{\infty, \infty}^{-1}}^{-1}\right) d \tau\right) .
\end{aligned}
$$

Taking the inner product of $(1.1)_{1}$ with $-\Delta u,(1.1)_{2}$ with $-\Delta \omega$ in $L^{2}\left(\mathbb{R}^{3}\right)$, and adding the resulting equations together, we obtain

$$
\begin{aligned}
& \frac{1}{2} \frac{d}{d t}\left(\|\nabla u(\cdot, t)\|_{L^{2}}^{2}+\|\nabla \omega(\cdot, t)\|_{L^{2}}^{2}\right)+\|\Delta u\|_{L^{2}}^{2}+\|\Delta \omega\|_{L^{2}}^{2}+\|\nabla \nabla \cdot \omega\|_{L^{2}}^{2}+2\|\nabla \omega\|_{L^{2}}^{2} \\
& \quad=\int_{\mathbb{R}^{3}}(u \cdot \nabla) u \cdot \Delta u d x-\int_{\mathbb{R}^{3}}(\nabla \times \omega) \cdot \Delta u d x+\int_{\mathbb{R}^{3}}(u \cdot \nabla) \omega \cdot \Delta \omega d x-\int_{\mathbb{R}^{3}}(\nabla \times u) \cdot \Delta \omega d x
\end{aligned}
$$




$$
\begin{aligned}
& \leq\|u\|_{L^{4}}\|\nabla u\|_{L^{4}}\|\Delta u\|_{L^{2}}+\|\nabla \omega\|_{L^{2}}\|\Delta u\|_{L^{2}}+\|u\|_{L^{4}}\|\nabla \omega\|_{L^{4}}\|\Delta \omega\|_{L^{2}}+\|\nabla u\|_{L^{2}}\|\Delta \omega\|_{L^{2}} \\
& \leq C\|u\|_{L^{4}}^{\frac{6}{5}}\|\Delta u\|_{L^{2}}^{\frac{9}{5}}+\|\omega\|_{L^{2}}^{\frac{1}{2}}\|\Delta \omega\|_{L^{2}}^{\frac{1}{2}}\|\Delta u\|_{L^{2}}+\|u\|_{L^{4}}\|\omega\|_{L^{4}}^{\frac{1}{5}}\|\Delta \omega\|_{L^{2}}^{\frac{9}{5}}+\|u\|_{L^{2}}^{\frac{1}{2}}\|\Delta u\|_{L^{2}}^{\frac{1}{2}}\|\Delta \omega\|_{L^{2}} \\
& \leq C\|u\|_{L^{4}}^{12}+C\|\omega\|_{L^{2}}^{2}+C\left(\|u\|_{L^{4}}^{12}+\|\omega\|_{L^{4}}^{12}\right)+C\|u\|_{L^{2}}^{2}+\frac{1}{2}\left(\|\Delta u\|_{L^{2}}^{2}+\|\Delta \omega\|_{L^{2}}^{2}\right) \\
& \leq C\left(1+\|u\|_{L^{4}}^{12}+\|\omega\|_{L^{4}}^{12}\right)+\frac{1}{2}\left(\|\Delta u\|_{L^{2}}^{2}+\|\Delta \omega\|_{L^{2}}^{2}\right),
\end{aligned}
$$

where we have used the Gagliardo-Nirenberg inequalities:

$$
\|\nabla u\|_{L^{4}} \leq C\|u\|_{L^{4}}^{\frac{1}{5}}\|\Delta u\|_{L^{2}}^{\frac{4}{5}} \quad \text { and } \quad\|\nabla u\|_{L^{2}} \leq C\|u\|_{L^{2}}^{\frac{1}{2}}\|\Delta u\|_{L^{2}}^{\frac{1}{2}} .
$$

This yields

$$
\begin{aligned}
& \frac{d}{d t}\left(\|\nabla u(\cdot, t)\|_{L^{2}}^{2}+\|\nabla \omega(\cdot, t)\|_{L^{2}}^{2}\right)+\|\Delta u\|_{L^{2}}^{2}+\|\Delta \omega\|_{L^{2}}^{2}+\|\nabla \nabla \cdot \omega\|_{L^{2}}^{2}+2\|\nabla \omega\|_{L^{2}}^{2} \\
& \quad \leq C\left(1+\|u\|_{L^{4}}^{12}+\|\omega\|_{L^{4}}^{12}\right) .
\end{aligned}
$$

Integrating the above inequality over $(0, t)$, we have

$$
\begin{aligned}
& \|\nabla u(t)\|_{L^{2}}^{2}+\|\nabla \omega(t)\|_{L^{2}}^{2}+\int_{0}^{t}\left(\|\Delta u(\tau)\|_{L^{2}}^{2}+\|\Delta \omega(\tau)\|_{L^{2}}^{2}+\|\nabla \nabla \cdot \omega(\tau)\|_{L^{2}}^{2}+2\|\nabla \omega(\tau)\|_{L^{2}}^{2}\right) d \tau \\
& \leq\left\|\nabla u_{0}\right\|_{L^{2}}^{2}+\left\|\nabla \omega_{0}\right\|_{L^{2}}^{2}+C \int_{0}^{t}\left(1+\|u(\tau)\|_{L^{4}}^{12}+\|\omega(\tau)\|_{L^{4}}^{12}\right) d \tau .
\end{aligned}
$$

On the other hand, by a Sobolev embedding theorem $\dot{H}^{1}\left(\mathbb{R}^{3}\right) \hookrightarrow L^{6}\left(\mathbb{R}^{3}\right),(2.8)$ and (1.5), we obtain that

$$
\begin{aligned}
e+\|\pi(\cdot, t)\|_{L^{3}} & \leq e+C\|u(\cdot, t)\|_{L^{6}}^{2} \leq e+C\|\nabla u(\cdot, t)\|_{L^{2}}^{2} \\
& \leq e+C\left(\left\|\nabla u_{0}\right\|_{L^{2}}^{2}+\left\|\nabla \omega_{0}\right\|_{L^{2}}^{2}\right)+C \int_{0}^{t}\left(1+\|u(\cdot, \tau)\|_{L^{4}}^{12}\right) d \tau \\
& \leq e+C\left(\left\|\nabla u_{0}\right\|_{L^{2}}^{2}+\left\|\nabla \omega_{0}\right\|_{L^{2}}^{2}\right)+C(e+t) \sup _{0 \leq \tau \leq t}\left(1+\|u(\cdot, \tau)\|_{L^{4}}^{12}\right) \\
& \leq C\left(e+\left\|\nabla u_{0}\right\|_{L^{2}}^{2}+\left\|\nabla \omega_{0}\right\|_{L^{2}}^{2}\right)(e+t) \sup _{0 \leq \tau \leq t}\left(1+\|u(\cdot, \tau)\|_{L^{4}}^{12}\right) \\
& \leq C_{0}(e+t) \exp \left(C \int_{0}^{t}\left(1+\|\pi(\cdot, \tau)\|_{\dot{B}_{\infty, \infty}^{-1}}^{2}\right) d \tau\right)
\end{aligned}
$$

where the constant $C_{0}=C\left(e,\left\|\nabla u_{0}\right\|_{L^{2}},\left\|\nabla \omega_{0}\right\|_{L^{2}},\left\|u_{0}\right\|_{L^{4}},\left\|\omega_{0}\right\|_{L^{4}}\right)$. Using the fact that $L^{3}\left(\mathbb{R}^{3}\right) \subset \dot{B}_{\infty, \infty}^{-1}\left(\mathbb{R}^{3}\right)$, it follows that

$$
e+\|\pi(\cdot, t)\|_{\dot{B}_{\infty, \infty}^{-1}} \leq C(e+t) \exp \left(C \int_{0}^{t}\left(1+\|\pi(\cdot, \tau)\|_{\dot{B}_{\infty, \infty}^{-1}}^{2}\right) d \tau\right)
$$

Now, taking the logarithm on both sides of (2.10), we can conclude that

$$
\log \left(e+\|\pi(\cdot, t)\|_{\dot{B}_{\infty, \infty}^{-1}}\right) \leq \log (C(e+t))+C \int_{0}^{t}\left(1+\|\pi(\cdot, \tau)\|_{\dot{B}_{\infty, \infty}^{-1}}^{2}\right) d \tau .
$$

For simplicity, let

$$
\begin{gathered}
\mathcal{Z}(t)=\log \left(e+\|\pi(\cdot, t)\|_{\dot{B}_{\infty, \infty}^{-1}}\right) \\
\mathcal{E}(t)=\log (C(e+t))+C \int_{0}^{t}\left(1+\|\pi(\cdot, \tau)\|_{\dot{B}_{\infty, \infty}^{-1}}^{2}\right) d \tau
\end{gathered}
$$


with $\mathcal{E}(0)=\log (\mathrm{Ce})$. Then, the above inequality (2.11) implies that

$$
0<\mathcal{Z}(t) \leq \mathcal{E}(t)
$$

and we easily get

$$
(e+\mathcal{Z}(t)) \log (e+\mathcal{Z}(t)) \leq(e+\mathcal{E}(t)) \log (e+\mathcal{E}(t)) .
$$

On the other hand, we have

$$
\begin{aligned}
\frac{d}{d t} \log (e+\mathcal{E}(t)) & =\frac{1}{e+\mathcal{E}(t)}\left(\frac{1}{e+t}+C\left(1+\|\pi(\cdot, t)\|_{\dot{B}_{\infty, \infty}^{-1}}^{2}\right)\right) \\
& \leq \frac{1}{e^{2}}+C \frac{1+\|\pi(\cdot, t)\|_{\dot{B}_{\infty, \infty}^{-1}}^{2}}{e+\mathcal{E}(t)} \\
& =\frac{1}{e^{2}}+C \frac{1+\|\pi(\cdot, t)\|_{\dot{B}_{\infty, \infty}^{-1}}^{2}}{(e+\mathcal{E}(t)) \ln (e+\mathcal{E}(t))} \log (e+\mathcal{E}(t)) \\
& \leq \frac{1}{e^{2}}+C \frac{1+\|\pi(\cdot, t)\|_{\dot{B}_{\infty, \infty}^{-1}}^{2}}{(e+\mathcal{Z}(t)) \ln (e+\mathcal{Z}(t))} \log (e+\mathcal{E}(t))
\end{aligned}
$$

Applying the Gronwall inequality to $\log (e+\mathcal{E}(t))$, we find

$$
\begin{aligned}
& \log (e+\mathcal{E}(t)) \\
& \quad \leq \log (e+\mathcal{E}(0)) \exp \left(\frac{T}{e^{2}}+C \int_{0}^{t} \frac{1+\|\pi(\cdot, \tau)\|_{\dot{B}_{\infty, \infty}^{-1}}^{2}}{(e+\mathcal{Z}(\tau)) \log (e+\mathcal{Z}(\tau))} d \tau\right),
\end{aligned}
$$

which yields

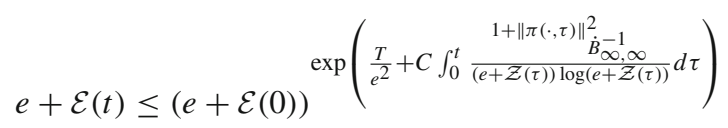

and from (2.12), we deduce that

$$
\int_{0}^{t}\|\pi(\cdot, \tau)\|_{\dot{B}_{\infty, \infty}^{-1}}^{2} d \tau \leq(e+\mathcal{E}(0))^{\exp \left(\frac{T}{e^{2}}+\frac{1}{C} \int_{0}^{t} \frac{1+\|\pi(\cdot, \tau)\|_{\dot{B}_{\infty, \infty}^{-1}}^{-1}}{(e+\mathcal{Z}(\tau)) \log (e+\mathcal{Z}(\tau))} d \tau\right)}<\infty .
$$

Hence by virtue of (2.7), (2.8) and (2.13), we conclude that

$$
(u, \omega) \in L^{\infty}\left((0, T) ; H^{1}\left(\mathbb{R}^{3}\right)\right) \cap L^{2}\left((0, T) ; H^{2}\left(\mathbb{R}^{3}\right)\right),
$$

which completes the proof of Theorem 1.1.

Acknowledgements The publication was prepared with the support of the "RUDN University Program 5100 ". Part of the work was carried out while S. Gala was a long-term visitor at the University of Catania. The hospitality of Catania University is graciously acknowledged. Research of M. Théra is supported by the Australian Research Council (ARC) grant DP160100854 and benefited from the support of the FMJH Program PGMO and from the support of EDF. The funding was provided by Gruppo Nazionale per 1'Analisi Matematica, la Probabilità e le loro Applicazioni (2019). 


\section{References}

1. Chen, J., Chen, Z.-M., Dong, B.-Q.: Uniform attractors of non-homogeneous micropolar fluid flows in non-smooth domains. Nonlinearity 20, 1619-1635 (2007)

2. Chen, Z.-M., Price, W.: Decay estimates of linearized micropolar fluid flows in $\mathbb{R}^{3}$ space with applications to $L^{3}$-strong solutions. Int. J. Eng. Sci. 44, 859-873 (2006)

3. Dong, B.-Q., Chen, Z.-M.: Regularity criteria of weak solutions to the three-dimensional micropolar flows. J. Math. Phys. 50, 103525 (2009)

4. Dong, B.-Q., Zhang, W.: On the regularity criterion for the 3D micropolar fluid flows in Besov spaces. Nonlinear Anal. 73, 2334-2341 (2010)

5. Dong, B.-Q., Jia, Y., Chen, Z.-M.: Pressure regularity criteria of the three-dimensional micropolar fluid flows. Math. Method Appl. Sci. 34, 595-606 (2011)

6. Eringen, A.: Theory of micropolar fluids. J. Math. Mech. 16, 1-18 (1966)

7. Gala, S.: On regularity criteria for the three-dimensional micropolar fluid equations in the critical MorreyCampanato space. Nonlinear Anal. 32, 2142-2150 (2011)

8. Gala, S.: A remark on the logarithmically improved regularity criterion for the micropolar fluid equations in terms of the pressure. Math. Method Appl. Sci. 34, 1945-1953 (2011)

9. Gala, S., Yan, J.: Two regularity criteria via the logarithm of the weak solutions to the micropolar fluid equations. J. Partial Differ. Eq. 25, 32-40 (2012)

10. Galdi, G., Rionero, S.: A note on the existence and uniqueness of solutions of micropolar fluid equations. Int. J. Eng. Sci. 14, 105-108 (1977)

11. Giga, Y.: Solutions for semilinear parabolic equations in Lp and regularity of weak solutions of the Navier-Stokes equations. J. Differ. Eq. 62, 186-212 (1986)

12. Guo, Z., Gala, S.: Remarks on logarithmical regularity criteria for the Navier-Stokes equations. J. Math. Phys. 52, 063503 (2011)

13. He, X., Gala, S.: Regularity criterion for weak solutions to the Navier-Stokes equations in terms of the pressure in the class $L^{2}\left(0, T ; B_{\infty, \infty}^{-1}\left(\mathbb{R}^{3}\right)\right)$. Nonlinear Anal. 12, 3602-3607 (2011)

14. Łukaszewicz, G.: Micropolar Fluids. Theory and Applications, Modeling and Simulation in Science, Engineering and Technology. Birkhä user, Boston (1999)

15. Meyer,Y., Gerard,P., Oru,F., Inégalités de Sobolev pr écisées : Séminaire Équations aux dérivées partielles (Polytechnique) (1996-1997), Exp. No. 4

16. Yuan, B.: On regularity criteria for weak solutions to the micropolar fluid equations in Lorentz space. Proc. Am. Math. Soc. 138, 2025-2036 (2010)

17. Zhang, W., Jia, Y., Dong, B.: Remarks on the regularity criterion of the 3D micropolar fluid flows in terms of the pressure. Appl. Math. Lett. 24, 199-203 (2011)

Publisher's Note Springer Nature remains neutral with regard to jurisdictional claims in published maps and institutional affiliations. 